Wozu noch Germanistik? 


\section{WOZU NOCH \\ GERMANISTIK?}

Wissenschaft - BerufKulturelle Praxis

Herausgegeben von Jürgen Förster, Eva Neuland und Gerhard Rupp

J.B. Metzlersche Verlagsbuchhandlung Stuttgart 
CIP-Titelaufnahme der Deutschen Bibliothek

Wozu noch Germanistik? : Wissenschaft - Beruf - kulturelle

Praxis / hrsg. von Jürgen Förster . . . - Stuttgart : Metzler, 1989

ISBN 978-3-476-00653-0

NE: Förster, Jürgen [Hrsg.]

ISBN 978-3-476-00653-0

ISBN 978-3-476-03271-3 (eBook)

DOI 10.1007/978-3-476-03271-3

Dieses Werk einschließlich aller seiner Teile ist urheberrechtlich geschützt. Jede Verwertung außerhalb der engen Grenzen des Urheberrechtsgesetzes ist ohne Zustimmung des Verlages unzulässig und strafbar.

Das gilt insbesondere für Vervielfältigungen, Übersetzungen, Mikroverfilmungen und die Einspeicherung und Verarbeitung in elektronischen Systemen.

(C) 1989 Springer-Verlag GmbH Deutschland

Ursprünglich erschienen bei J.B. Metzlersche Verlagsbuchhandlung und Carl Ernst Poeschel Verlag GmbH in Stuttgart 1989 


\section{Inhalt}

JÜRGEN FÖRSTER, EVA NEULAND, GERHARD RUPP

1 Wozu noch Germanistik?

Zur Aktualität einer alten Fragestellung

VOM ENDE DER GERMANISTIK UND IHRER ZUKUNFT BESTANDSAUFNAHMEN - EINBLICKE - AUSSICHTEN

CHRISTA BÜRGER

16 Literatur und Literaturwissenschaft

Ausgrenzungsgeschichten

HUBERT IVO

22 Blick zurück nach vorn

Zum Verhältnis von "Wissenschaftlichkeit" und "Praxisbezug" - am Beispiel der Professionalisierung der Sprachdidaktik

WERNER RÖCKE

37 Die Aktualität der Anfänge

Zur theoretischen und politischen Relevanz der frühen Germanistik

HANS PETER HERRMANN

50 Abschaffung der Geisteswissenschaften?

Standortbestimmung im aktuellen Streit zwischen Politik und Hochschulen

\section{GERMANISTIK IM GESELLSCHAFTLICHEN WANDEL AUFGABEN EINER ZUKÜNFTIGEN GERMANISTIK}

Sprache, Literatur und autonomes Handeln

ALBERT BREMERICH-VOS

68 Aspekte sprachlicher Bildung in der "Informationsgesellschaft" HEINZ W. GIESE

81 Die Fragmentierung von Handlungsautonomie im Zeichen von Sprachlosigkeit, kultureller Deprivierung und neuen Kommunikationstechniken

KLAUS-PETER KLEIN

93 Textverständlichkeit

Bedingungsanalysen und Vermittlungsmöglichkeiten

Sprache, Literatur und intersubjektive Verständigung

BIANKA MINTE-KÖNIG

105 Massenkommunikation und Neue Medien

LUDGER HOFFMANN/WERNER NOTHDURFT

118 Kommunikation und Kommunikationsprobleme in Institutionen 
Sprache, Literatur und kulturelle Rezeption und Produktion

RAIMUND KEMPER

133 Zur öffentlichen Bedeutung der Literarhistorie

HANS-JÜRGEN BACHORSKI

151 Ein Durchgraben zu den Wurzeln hin ...

Literarische Zeugnisse von vergangenen, alternativen und doch nicht obsoleten Welten

UTZ MAAS

166 Die Bedeutung der Schrift zur Vergewisserung der Lebensverhältnisse Perspektiven der kulturanalytischen Sprachwissenschaft

ERNEST W. B. HESS-LÜTTICH

176 Interkulturelle Kommunikation

Aneignung und Austausch kultureller Fremderfahrung

Sprache, Literatur und Aneignung und Verarbeitung gesellschaftlicher Wirklichkeit

HOLGER RUDLOFF

193 Die literarische Verarbeitung des Zeitbewußtseins

Der Beitrag der Literatur zur Sinnkonstitution an Beispielen der zeitgenössischen deutschen Literatur BERND SCHEFFER

207 Gesellschaftlicher Wandel nur durch kulturellen Wandel

Literaturwissenschaft zwischen Eigenwilligkeit und Konsens, zwischen Essay, Empirie und Anwendung

JÜRGEN LINK/ROLF PARR

224 Militarisierung der 'Spracher?

Zum Verhältnis von elementarer Literatur, Mediendiskursen und subjektiver Aufrüstung

Sprache, Literatur und personale Identitätsbildung

WINFRED KAMINSKI

239 Kinder- und Jugendliteratur, Leseerziehung und Ich-Entwicklung GISELA SCHOENTHAL

248 Sprachliche Ausdrucksformen sozialer Identität am Beispiel von geschlechtstypischem Sprachgebrauch

\section{GERMANISTIK ALS KULTURARBEIT BERICHTE ÜBER PROJEKTE}

HELMUT MÖRCHEN

262 Literatur in der Erwachsenenbildung BETTINA ROLFES

270 Von der Schreibbewegung zu einer möglichen Wissenschaft vom Schreiben 
HEINZ-HERMANN KRÜGER/WERNER THOLE

280 Jugendliche Selbstinszenierungen und soziokulturelle Phantasie Jugendkulturen und Jugendkulturarbeit im Zeitalter der Krise der Moderne

292 Hinweise zu den Autoren 